\title{
Impact of aqueous and organic extracts of Rhodobryum roseum on inhibition of fungal and bacterial growth
}

\section{Singh Shivom ${ }^{1}$, Rathore Kajal S. ${ }^{2} \bowtie$ and Khanna Dev Raj ${ }^{3}$}

Received: 28.01.2020

Revised: 23.03.2020

Accepted: 14.04.2020

\begin{abstract}
Mosses have been known for millennia and highly esteemed all over the world as the rich source of bioactive compounds. The research targets on evolution of microbicidal potentialities of Rhodobryum roseum (extract) used against selected fungus $(X$. oryzae pv oryzae, S. enteric, P. multocida and M. plutonius) and bacteria (R. solani, S. rolfsii, F. oxysporum and $T$. indica) to assay antimicrobial activity. Impact of aqueous and undertaken organic viz., ethanol, acetone, choloform, petroleum ether, methanol extract of $R$. roseum, at varied concentrations and at different time intervals were examined against the growth of bacteria and fungus. All the aqueous extracts were proved to be infective against all the tested pathogens. The antimicrobial potential of six extracts was screened against undertaken bacteria and fungi using micro broth dilution assay. Out of the six (diverse organic and aqueous) extract of $R$. roseum in ethanol and acetone showed maximum inhibitory activity in $S$. rolfsii with the MIC value of $5.00(\mu \mathrm{g} / \mathrm{ml})$, along with MFC value of $6.25(\mu \mathrm{g} / \mathrm{ml}) \mathrm{in}$ acetone extract and the value of $\mathrm{MBC}$ was recorded utmost in $X$. oryzae with value $3.00(\mu \mathrm{g} / \mathrm{ml})$ extracted in ethanol. Over all, the organic extract of $R$. roseum has potent antimicrobial activity and could be possible source of lead molecules considered for the future development of microbicidal agent.
\end{abstract}

Key Words: Rhodobryum roseum, moss, organic and aqueous extract, inhibitory concentration, microbicidal and pathogens

\section{Introduction}

In developing nations like India, microbes are considered as chief causative agents for many serious diseases and are of foremost concern by either private or authorized health care organizations. The financial emergency, low production and high cost of new generation antibiotics, inefficient community access to medicinal and pharmaceutical concern, along with the side effects caused by the synthetic drugs are some of the important aspects. To overcome these issues, research work in many countries is focusing to the vital character of medicinal plants and showing its contribution in wellbeing concern (Kumar et al., 2002). Therefore, in current scenario, rapid growth of medicinally significant multi resistant microbial trains of pathogens attract the researchers in all over world to discover the novel broad-spectrum antimicrobial agents.

Author's Address

${ }^{1}$ Department of Environmental Science, ITM University Gwalior, MP (India)

${ }^{2}$ Biotechnology Department, Government Kamla Raja Post Graduate College, Gwalior, MP (India)

${ }^{3}$ Department of Zoology and Environmental Science, Gurukula Kangri Vishwavidyalaya, Haridwwar,UK. (India)

E-mail.: drkajals101@gmail.com
Numerous herbs containing magnificent microbicidal potentialities have been accounted in many previous data (Dewanjee et al., 2007). Various researches have been performed with the extracts of number of plant species for the screening of their antimicrobial activity as well as for understanding the novel antimicrobial complexes, so that, they can be used for human welfare (Sengupta et al. 2008)).

Bryophytes (mosses, hornworts, and liverworts) are fascinating group of organisms found in all ecosystems of earth and carry numerous phytochemical constituents, therapeutic agents along with the secondary metabolites i.e. phenols, terpenoids, flavonoids, derivatives of benzoic acids and cinnamic acids, etc. However, due to its small size, inconspicuous place in the ecosystem and lack of commercial value, they are treated as hidden treasure for the world. Thus, the work presented reveals the incredible significance of these small creatures (mosses) and characterize the microbicidal potentialities of the undertaken moss extracts i.e. invitro activites of $R$. roseum against economically important crop pathogens. 


\section{Material and Methods Moss Sampling}

Moss was collected from walls, roofs approximately 1.5 meter above ground height and natural rocks which are devoid of no overhanging flora or tree coverings. The estimation was done in different sites (urban, sub-urban and rural) of Kumaon hills (Nainital, Almora, Jageshwar and Mukteshwar) for collecting only mature green plants, while the dead and dried plants (pale in colour) were rejected.

Moss Identification

Bryophytes were collected from different catchment sites of Kumaon hills during 2009-2010. The collected samples were kept in clean plastic bags and were brought to the laboratory for identification with the help of available taxonomical literatures fig.1 (Chopra, 1975; Smith, 1978; Gangulee, 1969; Saxena et al., 2008).

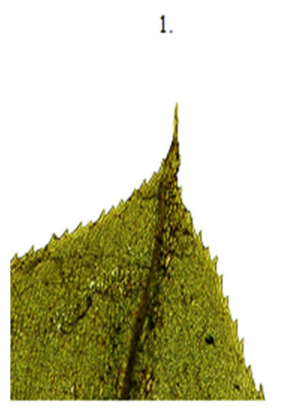

4.

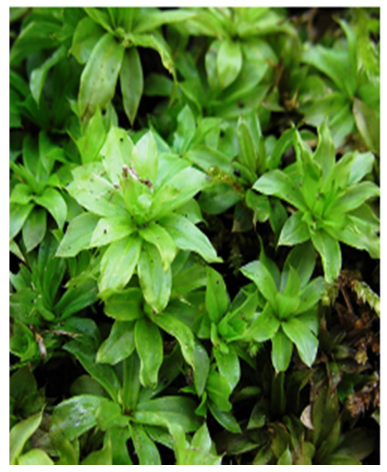

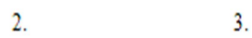
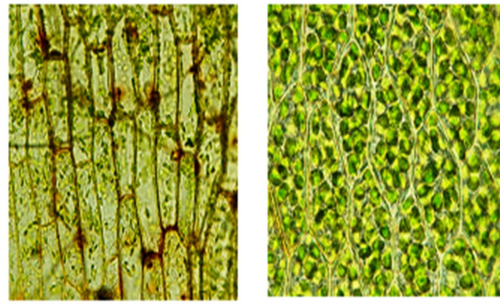

5.

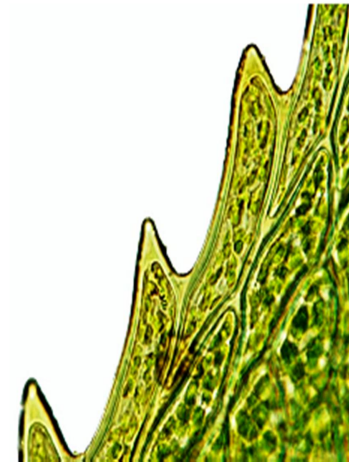

Figure.1 : Rhodobryum roseum (Hedw.) Limpr.

1. Leaves, 2. Basal cells, 3. Midleaf cells, 4. Whole plant 5 Margin cells

\section{Moss Extract}

The collected moss samples were cleaned carefully to remove the debris and dead material attached and afterword washed in running water and at last with distilled water. The washed samples were dried in shade and then finely powdered (100 g) using grinder. The obtained powder of moss was then extracted by mean of Soxhlet apparatus along with $500 \mathrm{ml}$ of different organic solvents (petroleum ether, chloroform, acetone, ethanol and methanol). The obtained extracts were filtered using muslin cloth and were left at room temperature for complete evaporation. In last step different concentrations $(40,200,500$ and $1000 \mu \mathrm{g} / \mathrm{mL})$ of crude extract were prepared for experimentation.

\section{Examination of Antimicrobial Activity}

Streptomycin and ampicillin antibiotics were used as positive controls to antibacterial activity $(X$. oryzae pv. oryzae, S. enterica, P. multocida, and $M$. plutonius). Antimicrobial activity was estimated by using disc diffusion method. The bacterial nutrient agar plates were treated with organic extracts (40 $\mu \mathrm{L} /$ disc) the nutrient and then incubated for $24 \mathrm{hrs}$ at $37 \pm 2^{\circ} \mathrm{C}$ (Basri and Fan, 2005). Antibacterial activity of the moss extracts was done by measuring the zone of inhibition (ZI) in mm against studied bacterial strains.

To check the fungal activity ( $F$. oxysporum $f$. $s p$. Lycopersci, $T$. indica, $s$. rolfsii and $R$. solanii) fungicide "Chloramine $\mathrm{T}$ " was used as positive control. Plates of PDA (Potato Dextrose Agar) were prepared under aseptic conditions and kept at $28 \pm$ $2^{0} \mathrm{C}$ for $72 \mathrm{hrs}$ for solidification. Four discs (two treated with moss extract + two controls) were kept in each petri plate with the test fungi.

\section{Calculation of MIC (Minimum Inhibitory Concentration) and $\mathrm{MBC}$ (Minimum Bactericidal Concentration)/MFC (Minimum Fungicidal Concentration)}

Janovska and his co workers (2003) carried out micro broth dilution assay to screen out the inhibitory and bacterial/fungicidal concentration of organic extracts. Fresh diluents were prepared for bacteria (nutrient broth) and fungi (potato dextrose agar) and the fresh and revived culture of untaken microorganisms were diluted in broth about 100 folds (100 $\mu \mathrm{L}$ microorganism in $10 \mathrm{~mL}$ broth).

CFU (Colony Forming Unit) was measured at optical density (OD) $620 \mathrm{~nm}$ (UV-visible spectrophotometer) and was observed 1 x 106 $\mathrm{CFU} / \mathrm{mL}$ (bacteria) and $1 \times 109 \mathrm{CFU} / \mathrm{mL}$ (fungi). The inoculated tubes of bacteria were incubated for 
$24 \mathrm{hrs}$ at $37^{\circ} \mathrm{C}$ and that of fungi for $72 \mathrm{hrs}$ at $28^{\circ} \mathrm{C}$ and later OD was measured at $620 \mathrm{~nm}$ via UV visible spectrophotometer.The lowest concentration that inhibited visible growth of tested organisms was recorded as MIC, and that caused no visible microbial growth was considered as MBC.

\section{Statistical Analysis}

The experimental data was evaluated statistical by using ANOVA. The study was based on three replicates to examine the effect of treatments separately and were depicted as mean \pm SE. Level of significance at $\mathrm{P}<0.05$ among undertaken microorganisms and different extracts was done by using JMP 5.0, SAS Institute, Cary, NC, USA. Furthermore, the mean of treatments were distinguished by using Turkey HSD test.

\section{Results and Discussion}

\section{Effect of extracts of $R$. roseum were examined} against the pathogenic plant fungus

Fusarium oxysporum results depict that, moss extract with chloramine $\mathrm{T}$ (positive control), at 1000 and $500 \mu \mathrm{g} \mathrm{ml}^{-1}$ conc. was showing significant higher values except that in methanol extract. However, out of the all undertaken extracts (aqueous and organic) of $R$. roseum, percent inhibition was maximum at $24 \mathrm{hrs}$ (38.37) in acetone extract at $1000 \mu \mathrm{g} \mathrm{ml}^{-1}$ conc. and minimum at $72 \mathrm{hrs}(8.29)$ in methanol extract at $40 \mu \mathrm{g} \mathrm{ml}^{-1}$ conc. (Table 1). Interestingly, aqueous extract of $R$. roseum had no activity against $F$. oxysporum at all conc. and time intervals. In addition, undertaken extracts with chloramine $\mathrm{T}$ (positive control) shows significantly higher values at all time interval with 100,500 and $200 \mu \mathrm{g} \mathrm{ml}^{-1}$ conc. in Tilletia indica out of the 6 extracts (aqueous and organic) of $R$. roseum, percent inhibition was maximum at $24 \mathrm{hrs}$ (34.27) with chloroform extract at $1000 \mu \mathrm{g} \mathrm{ml}^{-1}$ conc. and minimum at $72 \mathrm{hrs}$ (9.34) in acetone extract at $40 \mu \mathrm{g} \mathrm{ml}^{-1}$ conc. (Table 2). Interestingly, aqueous extract of $R$. roseum had no activity against $T$. indica at all conc. and time intervals.

Furthermore, results depict significantly higher inhibition growth in petroleum ether, methanol and acetone in 1000,500 and $200 \mu \mathrm{g} \mathrm{ml}^{-1}$ conc. over to chloramine T. However, in case of Sclerotium rolfsii out of the all extracts (aqueous and organic) of $R$. roseum, the maximum percent inhibition was observed in methanol extract (32.23) at $72 \mathrm{hrs}$ in $1000 \mu \mathrm{g} \mathrm{ml}^{-1}$ conc., while, the minimum percent inhibition was observed in petroleum ether extract (8.19) at $72 \mathrm{hrs}$ in $40 \mu \mathrm{g} \mathrm{ml}^{-1}$ conc. (Table 3). Here also, aqueous extract of $R$. roseum had no activity against $S$. rolfsii at all conc. and time intervals.

Nevertheless, for Rhizoctonia solani out of the 6 extracts (aqueous and organic) of $R$. roseum, the maximum percent inhibition was observed in acetone extract $(24.21)$ at $72 \mathrm{hrs}$ in $1000 \mu \mathrm{g} \mathrm{ml}^{-1}$ conc., whereas, the minimum percent inhibition was observed in chloroform extract (3.09) at $24 \mathrm{hrs}$ in $200 \mu \mathrm{g} \mathrm{ml}{ }^{-1}$ conc. (Table 4). Interestingly, aqueous, extract of $R$. roseum had no activity against $R$. solani at all conc. and time intervals. On doing comparison between all extracts and chloramine $\mathrm{T}$ (positive control), results indicate, significantly higher values in methanol at 24 and 48 hrs in $1000 \mu \mathrm{g} \mathrm{ml}^{-1}$ conc.

According to Mekuria et al., (1999) and Mewari et al., (2007) bryophyte as a new source of antifungal substance in crop protection. Eighteen bryophyte species were screened for antifungal nature of their ethanolic extracts. B. trilobata, D. albicans, S. quinquefarium, $D$. denudatum and $H$. spledens caused the greatest inhibition of mycelial growth of $B$. cinerea and $A$. solani. Also, antimicrobial activities ethanol and petroleum ether extracts of mosses, R. vagans Jaeg and E. plicatus C. Muell against two fungal pathogen, $B$. sorokiniana and $F$. solani and found both the extracts of mosses were effective for the tested fungi. Mekuria (2005) also reported the antifungal activity of 20 other bryophytes both under in vitro and in vivo conditions. The bryophytes showed the great antifungal capacity against phytopathogens, various researches revealed that the aqueous, methanolic extract of $R$. gangetica and mosses $C$. fontinaloides, A. viticulosus, T. tamarsicinum, E. striatum, $I$. alopecuroides and $P$. formosum and liverworts $P$. platyphylla and $S$. anemorea had antifungal activity against plant pathogenic fungi (Viz. $B$. dothidea, $B$. cinerea, $P$. viticola, $C$. acutatum, $M$. laxa and Calosphaeria sp.). The fungus toxicity of the aqueous extract was precised by inhibition of percent spore germination and hyphal length using 
Table 1: Percent inhibition in the growth of Fusarium oxysporum f.sp. lycopersici with different extract of $R$. roseum.

\begin{tabular}{|c|c|c|c|c|c|c|c|c|c|c|c|c|}
\hline \multirow{4}{*}{$\begin{array}{c}\text { Nature } \\
\text { of } \\
\text { extract }\end{array}$} & \multicolumn{12}{|c|}{ Concentration $\left(\mu \mathrm{g} \mathrm{ml}^{-1}\right)$} \\
\hline & \multicolumn{12}{|c|}{ Time (hrs.) } \\
\hline & \multicolumn{3}{|c|}{1000} & \multicolumn{3}{|c|}{500} & \multicolumn{3}{|c|}{200} & \multicolumn{3}{|c|}{40} \\
\hline & 24 & 48 & 72 & 24 & 48 & 72 & 24 & 48 & 72 & 24 & 48 & 72 \\
\hline \multirow[t]{2}{*}{ Petroleum ether } & $35.34^{\mathrm{c}}$ & $33.32^{\mathrm{a}}$ & $30.35^{\mathrm{a}}$ & $24.22^{\mathrm{a}}$ & $27.28^{\mathrm{a}}$ & $26.35^{\mathrm{a}}$ & $23.25^{\mathrm{a}}$ & $19.31^{\mathrm{a}}$ & $14.20^{\mathrm{a}}$ & $15.32^{\mathrm{a}}$ & $17.35^{\mathrm{d}}$ & $14.27^{\mathrm{b}}$ \\
\hline & \pm 0.27 & \pm 0.25 & \pm 0.27 & \pm 0.25 & \pm 0.20 & \pm 0.24 & \pm 0.16 & \pm 0.18 & \pm 0.23 & \pm 0.24 & \pm 0.26 & \pm 0.17 \\
\hline \multirow[t]{2}{*}{ Methanol } & $17.28^{\mathrm{d}}$ & $16.31^{b}$ & $15.33^{b}$ & $15.29^{b}$ & $16.34^{b}$ & $13.28^{\mathrm{b}}$ & $13.24^{b}$ & $12.25^{\mathrm{b}}$ & $10.36^{b}$ & $11.38^{b}$ & $9.30^{c}$ & $8.29^{\mathrm{d}}$ \\
\hline & \pm 0.16 & \pm 0.21 & \pm 0.25 & \pm 0.04 & \pm 0.24 & \pm 0.21 & \pm 0.29 & \pm 0.26 & \pm 0.34 & \pm 0.25 & \pm 0.22 & \pm 0.23 \\
\hline \multirow[t]{2}{*}{ Chloroform } & $30.30^{\mathrm{b}}$ & $32.34^{\mathrm{c}}$ & $33.48^{\mathrm{c}}$ & $30.25^{\mathrm{c}}$ & $28.23^{\mathrm{c}}$ & $25.25^{\mathrm{c}}$ & $25.33^{\mathrm{c}}$ & $23.34^{\mathrm{c}}$ & $20.20^{\mathrm{c}}$ & $22.20^{\mathrm{c}}$ & $19.31^{\mathrm{a}}$ & $15.26^{\mathrm{a}}$ \\
\hline & \pm 0.22 & \pm 0.21 & \pm 0.36 & \pm 0.16 & \pm 0.18 & \pm 0.16 & \pm 0.28 & \pm 0.32 & \pm 0.25 & \pm 0.22 & \pm 0.17 & \pm 0.17 \\
\hline Ethanol & 0.0 & 0.0 & 0.0 & 0.0 & 0.0 & 0.0 & 0.0 & 0.0 & 0.0 & 0.0 & 0.0 & 0.0 \\
\hline \multirow[t]{2}{*}{ Acetone } & $38.37^{\mathrm{a}}$ & $36.28^{\mathrm{d}}$ & $32.29^{\mathrm{d}}$ & $32.25^{\mathrm{d}}$ & $33.21^{\mathrm{d}}$ & $30.26^{\mathrm{d}}$ & $25.55^{\mathrm{d}}$ & $28.38^{\mathrm{d}}$ & $26.25^{d}$ & $18.35^{\mathrm{d}}$ & $16.35^{\mathrm{b}}$ & $14.23^{b}$ \\
\hline & \pm 0.22 & \pm 0.09 & \pm 0.21 & \pm 0.31 & \pm 0.25 & \pm 0.18 & \pm 0.16 & \pm 0.25 & \pm 0.13 & \pm 0.21 & \pm 0.21 & \pm 0.24 \\
\hline Water & 0.0 & 0.0 & 0.0 & 0.0 & 0.0 & 0.0 & 0.0 & 0.0 & 0.0 & 0.0 & 0.0 & 0.0 \\
\hline \multirow[t]{2}{*}{ Chloramine $\mathrm{T}$} & $21.34^{\mathrm{e}}$ & $25.33^{\mathrm{e}}$ & $26.24^{\mathrm{e}}$ & $23.38^{\mathrm{e}}$ & $21.32^{\mathrm{e}}$ & $24.39^{\mathrm{f}}$ & $22.30^{\mathrm{e}}$ & $20.28^{\mathrm{e}}$ & $18.42^{\mathrm{e}}$ & $19.36^{\mathrm{e}}$ & $16.17^{b}$ & $13.34^{\mathrm{c}}$ \\
\hline & \pm 0.19 & \pm 0.29 & \pm 0.15 & \pm 0.27 & \pm 0.24 & \pm 0.32 & \pm 0.22 & \pm 0.36 & \pm 0.19 & \pm 0.25 & \pm 0.19 & \pm 0.22 \\
\hline
\end{tabular}

Table 2: Percent inhibition in the growth of Tilletia indica with different extract of $R$. roseum.

\begin{tabular}{|c|c|c|c|c|c|c|c|c|c|c|c|c|}
\hline \multirow{4}{*}{$\begin{array}{c}\text { Nature } \\
\text { of } \\
\text { extract }\end{array}$} & \multicolumn{12}{|c|}{ Concentration $\left(\mu \mathrm{g} \mathrm{ml}^{-1}\right)$} \\
\hline & \multicolumn{12}{|c|}{$\begin{array}{l}\text { Time (hrs.) } \\
\end{array}$} \\
\hline & \multicolumn{3}{|c|}{1000} & \multicolumn{3}{|c|}{500} & \multicolumn{3}{|c|}{200} & \multicolumn{3}{|c|}{40} \\
\hline & 24 & 48 & 72 & 24 & 48 & 72 & 24 & 48 & 72 & 24 & 48 & 72 \\
\hline Petroleum ether & $26.12^{\mathrm{b}} \pm 0.11$ & $\begin{array}{l}24.29^{\mathrm{e}} \\
\pm 0.29\end{array}$ & $\begin{array}{l}22.25^{\mathrm{b}} \\
\pm 0.23\end{array}$ & $\begin{array}{l}23.24^{\mathrm{c}} \\
\pm 0.18\end{array}$ & $\begin{array}{l}20.46^{\mathrm{d}} \\
\pm 0.12\end{array}$ & $\begin{array}{l}17.42^{\mathrm{e}} \\
\pm 0.36\end{array}$ & $\begin{array}{c}20.37^{\mathrm{d}} \\
\pm 0.26\end{array}$ & $\begin{array}{l}16.42^{\mathrm{e}} \\
\pm 0.46\end{array}$ & $\begin{array}{l}16.11^{\mathrm{d}} \\
\pm 0.16\end{array}$ & $\begin{array}{l}15.35^{\mathrm{c}} \\
\pm 0.19\end{array}$ & $\begin{array}{l}12.18^{\mathrm{d}} \\
\pm 0.30\end{array}$ & $\begin{array}{l}11.44^{\mathrm{c}} \\
\pm 0.37\end{array}$ \\
\hline Methanol & $25.29^{\mathrm{c}} \pm 0.29$ & $\begin{array}{l}23.26^{\mathrm{d}} \\
\pm 0.15\end{array}$ & $\begin{array}{l}22.26^{b} \\
\pm 0.17\end{array}$ & $\begin{array}{l}23.32^{\mathrm{c}} \\
\pm 0.19\end{array}$ & $\begin{array}{l}22.33^{\mathrm{c}} \\
\pm 0.29\end{array}$ & $\begin{array}{l}20.31^{\mathrm{d}} \\
\pm 0.21\end{array}$ & $\begin{array}{c}20.27^{\mathrm{d}} \\
\pm 0.19\end{array}$ & $\begin{array}{l}18.27^{\mathrm{c}} \\
\pm 0.17\end{array}$ & $\begin{array}{l}15.25^{\mathrm{e}} \\
\pm 0.15\end{array}$ & $\begin{array}{l}18.30^{b} \\
\pm 0.24\end{array}$ & $\begin{array}{l}15.34^{b} \\
\pm 0.27\end{array}$ & $\begin{array}{c}12.30^{\mathrm{b}} \\
\pm 0.20\end{array}$ \\
\hline Chloroform & $34.27^{\mathrm{a}} \pm 0.25$ & $\begin{array}{c}30.29^{\mathrm{a}} \\
\pm 0.19\end{array}$ & $\begin{array}{l}28.52^{\mathrm{a}} \\
\pm 0.28\end{array}$ & $\begin{array}{c}26.36^{\mathrm{a}} \\
\pm 0.35\end{array}$ & $\begin{array}{l}25.33^{\mathrm{a}} \\
\pm 0.29\end{array}$ & $\begin{array}{l}23.37^{b} \\
\pm 0.34\end{array}$ & $\begin{array}{c}25.42^{\mathrm{a}} \\
\pm 0.18\end{array}$ & $\begin{array}{c}23.26^{\mathrm{a}} \\
\pm 0.24\end{array}$ & $\begin{array}{l}20.51^{\mathrm{a}} \\
\pm 0.39\end{array}$ & $\begin{array}{l}13.35^{\mathrm{d}} \\
\pm 0.18\end{array}$ & $\begin{array}{l}10.30^{\mathrm{e}} \\
\pm 0.20\end{array}$ & $9.36^{\mathrm{d}} \pm 0.26$ \\
\hline Ethanol & $23.38^{\mathrm{d}} \pm 0.37$ & $\begin{array}{l}25.34^{\mathrm{c}} \\
\pm 0.22\end{array}$ & $\begin{array}{l}22.32^{\mathrm{b}} \\
\pm 0.19\end{array}$ & $\begin{array}{l}26.42^{\mathrm{a}} \\
\pm 0.45\end{array}$ & $\begin{array}{l}23.18^{b} \\
\pm 0.21\end{array}$ & $\begin{array}{l}21.35^{\mathrm{c}} \\
\pm 0.31\end{array}$ & $\begin{array}{l}24.38^{b} \\
\pm 0.25\end{array}$ & $\begin{array}{l}19.52^{b} \\
\pm 0.37\end{array}$ & $\begin{array}{l}18.29^{c} \\
\pm 0.21\end{array}$ & $\begin{array}{l}15.44^{\mathrm{c}} \\
\pm 0.35\end{array}$ & $\begin{array}{l}12.48^{\mathrm{d}} \\
\pm 0.35\end{array}$ & $\begin{array}{l}11.39^{\mathrm{c}} \\
\pm 0.34\end{array}$ \\
\hline Acetone & $26.44^{b} \pm 0.28$ & $\begin{array}{l}25.25^{\mathrm{c}} \\
\pm 0.13\end{array}$ & $\begin{array}{l}23.29^{c} \\
\pm 0.22\end{array}$ & $\begin{array}{c}24.28^{b} \\
\pm 0.18\end{array}$ & $\begin{array}{l}20.26^{\mathrm{d}} \\
\pm 0.17\end{array}$ & $\begin{array}{l}17.30^{\mathrm{e}} \\
\pm 0.23\end{array}$ & $\begin{array}{l}20.37^{\mathrm{d}} \\
\pm 0.26\end{array}$ & $\begin{array}{l}18.40^{\mathrm{c}} \\
\pm 0.19\end{array}$ & $\begin{array}{l}15.31^{\mathrm{e}} \\
\pm 0.17\end{array}$ & $\begin{array}{l}15.35^{\mathrm{c}} \\
\pm 0.18\end{array}$ & $\begin{array}{l}13.25^{\mathrm{c}} \\
\pm 0.24\end{array}$ & $\begin{array}{l}9.346^{\mathrm{d}} \\
\pm 0.26\end{array}$ \\
\hline Water & 0.0 & 0.0 & 0.0 & 0.0 & 0.0 & 0.0 & 0.0 & 0.0 & 0.0 & 0.0 & 0.0 & 0.0 \\
\hline Chloramine $\mathrm{T}$ & $\begin{array}{l}25.31^{\mathrm{c}} \\
\pm 0.25\end{array}$ & $\begin{array}{l}28.26^{b} \\
\pm 0.18\end{array}$ & $\begin{array}{c}23.27^{\mathrm{c}} \\
\pm 0.18\end{array}$ & $\begin{array}{l}22.25^{\mathrm{d}} \\
\pm 0.15\end{array}$ & $\begin{array}{l}20.27^{\mathrm{d}} \\
\pm 0.18\end{array}$ & $\begin{array}{l}24.15^{\mathrm{a}} \\
\pm 0.17\end{array}$ & $\begin{array}{l}21.32^{\mathrm{c}} \\
\pm 0.19\end{array}$ & $\begin{array}{l}17.48^{\mathrm{d}} \\
\pm 0.39\end{array}$ & $\begin{array}{l}19.32^{b} \\
\pm 0.16\end{array}$ & $\begin{array}{l}20.33^{\mathrm{a}} \\
\pm 0.18\end{array}$ & $\begin{array}{l}16.53^{\mathrm{a}} \\
\pm 0.28\end{array}$ & $\begin{array}{l}14.30^{\mathrm{a}} \\
\pm 0.24\end{array}$ \\
\hline
\end{tabular}

*Values are represented as mean $\pm \mathrm{SD}$.

*The treatment means were separated using Tukey HSD at $0.05 \%$ probability level.

* Level not connected by same letter are significantly different in vertical columns. 
Impact of aqueous and organic extracts of Rhodobryum roseum

Table 3: Percent inhibition in the growth of Sclerotium rolfsii with different extracts of $R$. roseum.

\begin{tabular}{|c|c|c|c|c|c|c|c|c|c|c|c|c|}
\hline \multirow{4}{*}{$\begin{array}{c}\text { Nature } \\
\text { of } \\
\text { extract }\end{array}$} & \multicolumn{12}{|c|}{ Concentration $\left(\mu \mathrm{g} \mathrm{ml}^{-1}\right)$} \\
\hline & \multicolumn{12}{|c|}{ Time (hrs.) } \\
\hline & \multicolumn{3}{|c|}{1000} & \multicolumn{3}{|c|}{500} & \multicolumn{3}{|c|}{200} & \multicolumn{3}{|c|}{40} \\
\hline & 24 & 48 & 72 & 24 & 48 & 72 & 24 & 48 & 72 & 24 & 48 & 72 \\
\hline Petroleum ether & $\begin{array}{l}21.46^{\mathrm{b}} \\
\pm 0.37\end{array}$ & $\begin{array}{l}24.65^{\mathrm{a}} \\
\pm 0.30\end{array}$ & $\begin{array}{l}30.29^{\mathrm{a}} \\
\pm 0.32\end{array}$ & $\begin{array}{l}14.47^{\mathrm{a}} \\
\pm 0.15\end{array}$ & $\begin{array}{l}25.14^{\mathrm{a}} \\
\pm 0.11\end{array}$ & $\begin{array}{l}27.28^{\mathrm{a}} \\
\pm 0.28\end{array}$ & $\begin{array}{l}21.25^{\mathrm{a}} \\
\pm 0.31\end{array}$ & $\begin{array}{l}22.24^{\mathrm{a}} \\
\pm 0.25\end{array}$ & $\begin{array}{c}23.107^{\mathrm{a}} \\
\pm 0.11\end{array}$ & $\begin{array}{c}8.28^{b} \\
\pm 0.46\end{array}$ & $\begin{array}{l}8.30^{\mathrm{b}} \\
\pm 0.48\end{array}$ & $\begin{array}{c}8.19^{b} \\
\pm 0.23\end{array}$ \\
\hline Methanol & $\begin{array}{l}24.47^{\mathrm{a}} \\
\pm 0.43 \\
\end{array}$ & $\begin{array}{l}26.35^{\mathrm{b}} \\
\pm 0.36\end{array}$ & $\begin{array}{l}32.23^{\mathrm{b}} \\
\pm 0.37\end{array}$ & $\begin{array}{l}15.33^{\mathrm{b}} \\
\pm 0.27\end{array}$ & $\begin{array}{l}27.26^{b} \\
\pm 0.21\end{array}$ & $\begin{array}{c}28.28^{\mathrm{b}} \pm \\
0.30\end{array}$ & $\begin{array}{l}24.31^{b} \\
\pm 0.25\end{array}$ & $\begin{array}{l}23.34^{b} \\
\pm 0.20\end{array}$ & $\begin{array}{c}24.22^{\mathrm{b}} \\
\pm 0.30\end{array}$ & $\begin{array}{c}8.69^{b} \\
\pm 0.29 \\
\end{array}$ & $\begin{array}{c}8.32^{\mathrm{b}} \\
\pm 0.46\end{array}$ & $\begin{array}{l}8.36^{\mathrm{b}} \\
\pm 0.21\end{array}$ \\
\hline Chloroform & 0.0 & 0.0 & 0.0 & 0.0 & 0.0 & 0.0 & 0.0 & 0.0 & 0.0 & 0.0 & 0.0 & 0.0 \\
\hline Ethanol & 0.0 & 0.0 & 0.0 & 0.0 & 0.0 & 0.0 & 0.0 & 0.0 & 0.0 & 0.0 & 0.0 & 0.0 \\
\hline Acetone & $\begin{array}{l}15.33^{\mathrm{c}} \\
\pm 0.32\end{array}$ & $\begin{array}{l}18.28^{\mathrm{c}} \\
\pm 0.07\end{array}$ & $\begin{array}{l}16.21^{\mathrm{c}} \\
\pm 0.28\end{array}$ & $\begin{array}{l}29.45^{\mathrm{c}} \\
\pm 0.47\end{array}$ & $\begin{array}{l}31.01^{\mathrm{c}} \\
\pm 0.85\end{array}$ & $\begin{array}{l}30.32^{\mathrm{c}} \\
\pm 0.43\end{array}$ & $\begin{array}{l}23.55^{\mathrm{c}} \\
\pm 0.27\end{array}$ & $\begin{array}{l}21.33^{\mathrm{c}} \\
\pm 0.54\end{array}$ & $\begin{array}{l}21.11^{\mathrm{c}} \\
\pm 0.16\end{array}$ & $\begin{array}{l}8.31^{\mathrm{b}} \\
\pm 0.33 \\
\end{array}$ & $8.46^{b} \pm 0.38$ & $\begin{array}{l}8.26^{b} \\
\pm 0.34 \\
\end{array}$ \\
\hline Water & 0.0 & 0.0 & 0.0 & 0.0 & 0.0 & 0.0 & 0.0 & 0.0 & 0.0 & 0.0 & 0.0 & 0.0 \\
\hline Chloramine $\mathrm{T}$ & $\begin{array}{l}19.32^{\mathrm{d}} \\
\pm 0.06\end{array}$ & $\begin{array}{l}21.17^{\mathrm{d}} \\
\pm 0.27\end{array}$ & $\begin{array}{l}18.15^{\mathrm{d}} \\
\pm 0.22\end{array}$ & $\begin{array}{l}16.26^{\mathrm{d}} \\
\pm 0.25\end{array}$ & $\begin{array}{l}18.50^{\mathrm{d}} \\
\pm 0.35\end{array}$ & $\begin{array}{l}15.46^{\mathrm{d}} \\
\pm 0.33\end{array}$ & $\begin{array}{l}14.15^{\mathrm{d}} \\
\pm 0.23\end{array}$ & $\begin{array}{l}15.17^{\mathrm{d}} \\
\pm 0.27\end{array}$ & $\begin{array}{l}13.21^{\mathrm{d}} \\
\pm 0.34\end{array}$ & $\begin{array}{l}12.21^{\mathrm{a}} \\
\pm 0.46\end{array}$ & $\begin{array}{l}14.21^{\mathrm{a}} \\
\pm 0.40\end{array}$ & $\begin{array}{l}11.27^{\mathrm{a}} \\
\pm 0.36\end{array}$ \\
\hline
\end{tabular}

Table 4: Percent inhibition in the growth of Rhizoctonia solanii with different extract of $R$. roseum.

\begin{tabular}{|c|c|c|c|c|c|c|c|c|c|c|c|c|}
\hline \multirow{4}{*}{$\begin{array}{c}\text { Nature } \\
\text { of } \\
\text { extract }\end{array}$} & \multicolumn{12}{|c|}{ Concentration $\left(\mu \mathrm{g} \mathrm{ml}^{-1}\right)$} \\
\hline & \multicolumn{12}{|c|}{ Time (hrs.) } \\
\hline & \multicolumn{3}{|c|}{1000} & \multicolumn{3}{|c|}{500} & \multicolumn{3}{|c|}{200} & \multicolumn{3}{|c|}{40} \\
\hline & 24 & 48 & 72 & 24 & 48 & 72 & 24 & 48 & 72 & 24 & 48 & 72 \\
\hline Petroleum ether & $\begin{array}{c}7.15^{\mathrm{e}} \\
\pm 0.23\end{array}$ & $\begin{array}{l}5.63^{\mathrm{c}} \\
\pm 0.48\end{array}$ & $\begin{array}{c}3.25^{\mathrm{e}} \\
\pm 0.20\end{array}$ & $\begin{array}{c}6.50^{\mathrm{e}} \\
\pm 0.31\end{array}$ & $\begin{array}{c}7.24^{\mathrm{f}} \\
\pm 0.19\end{array}$ & $\begin{array}{l}3.05^{\mathrm{e}} \\
\pm 0.06\end{array}$ & $\begin{array}{l}15.26^{\mathrm{a}} \\
\pm 0.28\end{array}$ & $\begin{array}{c}20.28^{\mathrm{a}} \\
\pm 0.39\end{array}$ & 0.0 & $\begin{array}{c}9.28^{\mathrm{d}} \\
\pm 0.30\end{array}$ & $\begin{array}{c}3.23^{\mathrm{d}} \\
\pm 0.29\end{array}$ & 0.0 \\
\hline Methanol & $\begin{array}{l}15.21^{\mathrm{d}} \\
\pm 0.27\end{array}$ & $\begin{array}{l}15.46^{\mathrm{b}} \\
\pm 0.38 \\
\end{array}$ & $\begin{array}{l}15.12^{\mathrm{d}} \\
\pm 0.17 \\
\end{array}$ & $\begin{array}{l}11.42^{\mathrm{d}} \\
\pm 0.24 \\
\end{array}$ & $\begin{array}{l}14.46^{\mathrm{e}} \\
\pm 0.42 \\
\end{array}$ & $\begin{array}{c}5.66^{\mathrm{d}} \\
\pm 0.23 \\
\end{array}$ & $\begin{array}{l}11.29^{\mathrm{b}} \\
\pm 0.30\end{array}$ & $\begin{array}{c}6.08^{\mathrm{b}} \\
\pm 0.13 \\
\end{array}$ & 0.0 & 0.0 & 0.0 & 0.0 \\
\hline Chloroform & $\begin{array}{l}14.30^{\mathrm{c}} \\
\pm 0.22 \\
\end{array}$ & $\begin{array}{l}12.62^{\mathrm{d}} \\
\pm 0.36 \\
\end{array}$ & $\begin{array}{c}3.34^{\mathrm{e}} \\
\pm 0.29 \\
\end{array}$ & $\begin{array}{l}17.25^{\mathrm{c}} \\
\pm 0.25 \\
\end{array}$ & $\begin{array}{l}23.16^{\mathrm{d}} \\
\pm 0.13 \\
\end{array}$ & $\begin{array}{l}3.16^{\mathrm{e}} \\
\pm 0.13 \\
\end{array}$ & $\begin{array}{l}11.16^{b} \\
\pm 0.24\end{array}$ & $\begin{array}{c}3.09^{\mathrm{c}} \\
\pm 0.14 \\
\end{array}$ & 0.0 & $\begin{array}{l}14.33^{\mathrm{a}} \\
\pm 0.37\end{array}$ & $\begin{array}{c}3.09^{\mathrm{d}} \\
\pm 0.14\end{array}$ & 0.0 \\
\hline Ethanol & $\begin{array}{c}9.69^{\mathrm{b}} \\
\pm 0.21 \\
\end{array}$ & $\begin{array}{l}15.23^{b} \\
\pm 0.28 \\
\end{array}$ & $\begin{array}{l}16.48^{\mathrm{c}} \\
\pm 0.41 \\
\end{array}$ & $\begin{array}{l}10.15^{\mathrm{b}} \\
\pm 0.12\end{array}$ & $\begin{array}{l}18.12^{\mathrm{c}} \\
\pm 0.11 \\
\end{array}$ & $\begin{array}{l}12.16^{\mathrm{c}} \\
\pm 0.24\end{array}$ & $\begin{array}{l}9.35^{\mathrm{c}} \\
\pm 0.49 \\
\end{array}$ & $\begin{array}{l}14.26^{\mathrm{d}} \\
\pm 0.25 \\
\end{array}$ & $\begin{array}{l}13.31^{\mathrm{a}} \\
\pm 0.43 \\
\end{array}$ & $\begin{array}{l}8.33^{\mathrm{c}} \\
\pm 0.31 \\
\end{array}$ & $\begin{array}{l}6.25^{\mathrm{c}} \\
\pm 0.25 \\
\end{array}$ & $\begin{array}{r}6.18^{b} \\
\pm 0.21 \\
\end{array}$ \\
\hline Acetone & $\begin{array}{c}7.57^{\mathrm{e}} \\
\pm 0.33\end{array}$ & $\begin{array}{l}21.31^{\mathrm{a}} \\
\pm 0.28\end{array}$ & $\begin{array}{l}24.21^{b} \\
\pm 0.28\end{array}$ & $\begin{array}{c}6.44^{\mathrm{e}} \\
\pm 0.12\end{array}$ & $\begin{array}{l}19.28^{b} \\
\pm 0.25\end{array}$ & $\begin{array}{l}13.42^{\mathrm{b}} \\
\pm 0.33\end{array}$ & $\begin{array}{l}5.31^{\mathrm{d}} \\
\pm 0.24\end{array}$ & $\begin{array}{l}15.42^{\mathrm{e}} \\
\pm 0.25\end{array}$ & $\begin{array}{l}10.31^{b} \\
\pm 0.26\end{array}$ & $\begin{array}{l}4.39^{b} \\
\pm 0.31\end{array}$ & $\begin{array}{l}8.27^{\mathrm{b}} \\
\pm 0.35\end{array}$ & $\begin{array}{c}9.30^{c} \\
\pm 0.30\end{array}$ \\
\hline Water & 0.0 & 0.0 & 0.0 & 0.0 & 0.0 & 0.0 & 0.0 & 0.0 & 0.0 & 0.0 & 0.0 & 0.0 \\
\hline Chloramine $\mathrm{T}$ & $\begin{array}{l}12.37^{\mathrm{a}} \\
\pm 0.32 \\
\end{array}$ & $\begin{array}{l}14.24^{\mathrm{e}} \\
\pm 0.30 \\
\end{array}$ & $\begin{array}{l}19.27^{\mathrm{a}} \\
\pm 0.25 \\
\end{array}$ & $\begin{array}{c}16.23^{\mathrm{a}} \\
\pm .20 \\
\end{array}$ & $\begin{array}{l}17.26^{\mathrm{a}} \\
\pm 0.22 \\
\end{array}$ & $\begin{array}{l}14.21^{\mathrm{a}} \\
\pm 0.26 \\
\end{array}$ & $\begin{array}{l}15.25^{\mathrm{a}} \\
\pm 0.20 \\
\end{array}$ & $\begin{array}{l}13.28^{f} \\
\pm 0.29 \\
\end{array}$ & $\begin{array}{l}11.22^{\mathrm{c}} \\
\pm 0.36 \\
\end{array}$ & $\begin{array}{l}14.35^{\mathrm{a}} \\
\pm 0.22 \\
\end{array}$ & $\begin{array}{l}12.27^{\mathrm{a}} \\
\pm 0.32 \\
\end{array}$ & $\begin{array}{l}13.32^{\mathrm{a}} \\
\pm 0.28 \\
\end{array}$ \\
\hline
\end{tabular}

$*$ Values are represented as mean $\pm \mathrm{SD}$.

*The treatment means were separated using Tukey HSD at $0.05 \%$ probability level.

*Level not connected by same letter are significantly different in vertical columns. 
Singh et al.

Table 5 . Activity Index of the growth of Xanthomonas oryzae pv. oryzae with different extract of $R$. roseum

\begin{tabular}{|c|c|c|c|c|}
\hline \multirow{2}{*}{$\begin{array}{c}\begin{array}{c}\text { Nature } \\
\text { of } \\
\text { extract }\end{array} \\
\end{array}$} & \multicolumn{4}{|c|}{ Concentration $\left(\mu \mathrm{g} \mathrm{ml}^{-1}\right)$} \\
\hline & 1000 & 500 & 200 & 40 \\
\hline Petroleum ether & 0.0 & 0.0 & 0.0 & 0.0 \\
\hline Methanol & $\begin{array}{l}11.21^{\mathrm{c}} \\
\pm 0.32\end{array}$ & $\begin{array}{l}12.19^{\mathrm{c}} \\
\pm 0.29 \\
\end{array}$ & $\begin{array}{l}11.27^{\mathrm{c}} \\
\pm 0.24\end{array}$ & $\begin{array}{l}10.22^{\mathrm{c}} \\
\pm 0.26 \\
\end{array}$ \\
\hline Chloroform & 0.0 & 0.0 & 0.0 & 0.0 \\
\hline Ethanol & $\begin{array}{l}11.26^{\mathrm{c}} \\
\pm 0.34\end{array}$ & $\begin{array}{l}12.31^{\mathrm{c}} \\
\pm 0.28\end{array}$ & $\begin{array}{l}11.27^{\mathrm{c}} \\
\pm 0.27\end{array}$ & $\begin{array}{l}10.36^{\mathrm{c}} \\
\pm 0.32\end{array}$ \\
\hline Acetone & 0.0 & 0.0 & 0.0 & 0.0 \\
\hline Water & 0.0 & 0.0 & 0.0 & 0.0 \\
\hline Ampicillin & $\begin{array}{l}18.19^{b} \\
\pm 0.22\end{array}$ & $\begin{array}{l}15.24^{b} \\
\pm 0.22\end{array}$ & $\begin{array}{l}13.28^{b} \\
\pm 0.28\end{array}$ & $\begin{array}{l}11.21^{\mathrm{b}} \\
\pm 0.20\end{array}$ \\
\hline Streptomycin & $\begin{array}{c}20.29^{\mathrm{a}} \\
\pm 0.36\end{array}$ & $\begin{array}{l}18.26^{\mathrm{a}} \\
\pm 0.34\end{array}$ & $\begin{array}{l}16.20^{\mathrm{a}} \\
\pm 0.25\end{array}$ & $\begin{array}{l}13.26^{\mathrm{a}} \\
\pm 0.28 \\
\end{array}$ \\
\hline
\end{tabular}

Table 6: Activity Index of the growth of Melissococcus plutonius with different extract of $R$. roseum

\begin{tabular}{|c|c|c|c|c|}
\hline \multirow{2}{*}{$\begin{array}{c}\text { Nature } \\
\text { of } \\
\text { extract }\end{array}$} & \multicolumn{4}{|c|}{ Concentration $\left(\mu \mathrm{g} \mathrm{ml}^{-1}\right)$} \\
\hline & 1000 & 500 & 200 & 40 \\
\hline Petroleum ether & 0.0 & 0.0 & 0.0 & 0.0 \\
\hline Methanol & $\begin{array}{l}12.19^{\mathrm{c}} \\
\pm 0.23\end{array}$ & $\begin{array}{l}13.27^{\mathrm{a}} \\
\pm 0.28\end{array}$ & $\begin{array}{l}11.21^{\mathrm{a}} \\
\pm 0.33\end{array}$ & $\begin{array}{l}6.37^{\mathrm{d}} \\
\pm 0.27\end{array}$ \\
\hline Chloroform & 0.0 & 0.0 & 0.0 & 0.0 \\
\hline Ethanol & $\begin{array}{l}13.23^{b} \\
\pm 0.29\end{array}$ & $\begin{array}{l}12.30^{b} \\
\pm 0.24\end{array}$ & $\begin{array}{l}11.23^{\mathrm{a}} \\
\pm 0.28\end{array}$ & $\begin{array}{l}10.15^{\mathrm{a}} \\
\pm 0.14\end{array}$ \\
\hline Acetone & $\begin{array}{l}15.26^{\mathrm{a}} \\
\pm 0.25\end{array}$ & $\begin{array}{l}11.24^{\mathrm{c}} \\
\pm 0.29\end{array}$ & $\begin{array}{l}11.23^{\mathrm{a}} \\
\pm 0.28\end{array}$ & $\begin{array}{l}10.18^{\mathrm{a}} \\
\pm 0.15\end{array}$ \\
\hline Water & 0.0 & 0.0 & 0.0 & 0.0 \\
\hline Ampicillin & $\begin{array}{l}15.24^{\mathrm{a}} \\
\pm 0.28\end{array}$ & $\begin{array}{l}13.22^{\mathrm{a}} \\
\pm 0.29\end{array}$ & $\begin{array}{l}11.22^{\mathrm{a}} \\
\pm 0.26\end{array}$ & $\begin{array}{r}9.22^{\mathrm{b}} \\
\pm 0.27\end{array}$ \\
\hline Streptomycin & $\begin{array}{l}13.20^{b} \\
\pm 0.23\end{array}$ & $\begin{array}{l}11.31^{\mathrm{c}} \\
\pm 0.32\end{array}$ & $\begin{array}{l}10.27^{b} \\
\pm 0.32\end{array}$ & $\begin{array}{l}8.33^{\mathrm{c}} \\
\pm 0.26\end{array}$ \\
\hline
\end{tabular}

*Values are represented as mean \pm SD.

*The treatment means were separated using Tukey HSD at $0.05 \%$ probability level.

*Level not connected by same letter are significantly different in vertical columns. 
Table 7: Activity Index of the growth of Pasteurella multocida with different extract of $R$. roseum.

\begin{tabular}{|c|c|c|c|c|}
\hline \multirow{2}{*}{$\begin{array}{c}\text { Nature } \\
\text { of } \\
\text { extract }\end{array}$} & \multicolumn{4}{|c|}{ Concentration $\left(\mu \mathrm{g} \mathrm{ml}^{-1}\right)$} \\
\hline & 1000 & 500 & 200 & 40 \\
\hline Petroleum ether & 0.0 & 0.0 & 0.0 & 0.0 \\
\hline Methanol & 0.0 & 0.0 & 0.0 & 0.0 \\
\hline Chloroform & $\begin{array}{l}12.26^{\mathrm{d}} \\
\pm 0.27\end{array}$ & $\begin{array}{l}13.22^{b} \\
\pm 0.26\end{array}$ & $\begin{array}{l}11.23^{\mathrm{c}} \\
\pm 0.27\end{array}$ & $\begin{array}{l}10.23^{\mathrm{a}} \\
\pm 0.28\end{array}$ \\
\hline Ethanol & $\begin{array}{c}20.24^{\mathrm{a}} \\
\pm 0.30\end{array}$ & $\begin{array}{l}18.23^{\mathrm{a}} \\
\pm 0.28\end{array}$ & $\begin{array}{l}15.48^{\mathrm{a}} \\
\pm 0.15\end{array}$ & $\begin{array}{l}13.22^{\mathrm{b}} \\
\pm 0.27\end{array}$ \\
\hline Acetone & $\begin{array}{l}16.24^{b} \\
\pm 0.25\end{array}$ & $\begin{array}{l}13.26^{\mathrm{b}} \\
\pm 0.27\end{array}$ & $\begin{array}{l}14.25^{\mathrm{b}} \\
\pm 0.32\end{array}$ & $\begin{array}{l}12.20^{\mathrm{c}} \\
\pm 0.31\end{array}$ \\
\hline Water & 0.0 & 0.0 & 0.0 & 0.0 \\
\hline Ampicillin & $\begin{array}{l}15.27^{\mathrm{c}} \\
\pm 0.26\end{array}$ & $\begin{array}{l}13.30^{\mathrm{b}} \\
\pm 0.23\end{array}$ & $\begin{array}{l}11.24^{\mathrm{c}} \\
\pm 0.28\end{array}$ & $\begin{array}{l}9.20^{\mathrm{d}} \\
\pm .23 \\
\end{array}$ \\
\hline Streptomycin & $\begin{array}{l}15.26^{\mathrm{c}} \\
\pm 0.30\end{array}$ & $\begin{array}{l}12.26^{\mathrm{c}} \\
\pm 0.32\end{array}$ & $\begin{array}{l}10.23^{\mathrm{d}} \\
\pm 0.29\end{array}$ & $\begin{array}{c}8.29^{\mathrm{e}} \\
\pm 0.21\end{array}$ \\
\hline
\end{tabular}

Table 8: Activity Index of the growth of Salmonella enterica with different extract of $R$. roseum.

\begin{tabular}{|c|c|c|c|c|}
\hline \multirow{2}{*}{$\begin{array}{c}\text { Nature } \\
\text { of } \\
\text { extract }\end{array}$} & \multicolumn{4}{|c|}{ Concentration $\left(\mu \mathrm{g} \mathrm{ml}^{-1}\right)$} \\
\hline & 1000 & 500 & 200 & 40 \\
\hline Petroleum ether & 0.0 & 0.0 & 0.0 & 0.0 \\
\hline Methanol & 0.0 & 0.0 & 0.0 & 0.0 \\
\hline Chloroform & 0.0 & 0.0 & 0.0 & 0.0 \\
\hline Ethanol & $\begin{array}{l}11.28^{\mathrm{a}} \\
\pm 0.18\end{array}$ & $\begin{array}{l}15.21^{\mathrm{a}} \\
\pm 0.25\end{array}$ & $\begin{array}{l}11.22^{\mathrm{a}} \\
\pm 0.23\end{array}$ & $\begin{array}{l}13.22^{\mathrm{a}} \\
\pm 0.26\end{array}$ \\
\hline Acetone & $\begin{array}{l}13.19^{b} \\
\pm 0.16\end{array}$ & $\begin{array}{c}16.26^{\mathrm{b}} \\
\pm 0.19\end{array}$ & $\begin{array}{l}13.25^{\mathrm{b}} \\
\pm 0.21\end{array}$ & $\begin{array}{l}11.19^{\mathrm{b}} \\
\pm 0.22\end{array}$ \\
\hline Water & 0.0 & 0.0 & 0.0 & 0.0 \\
\hline Ampicillin & $\begin{array}{c}20.38^{\mathrm{c}} \\
\pm 0.24\end{array}$ & $\begin{array}{l}16.31^{b} \\
\pm 0.27\end{array}$ & $\begin{array}{l}13.28^{b} \\
\pm 0.28\end{array}$ & $\begin{array}{l}10.21^{\mathrm{c}} \\
\pm 0.24\end{array}$ \\
\hline Streptomycin & $\begin{array}{l}18.30^{\mathrm{d}} \\
\pm 0.31\end{array}$ & $\begin{array}{l}16.29^{b} \\
\pm 0.17\end{array}$ & $\begin{array}{l}13.28^{b} \\
\pm 0.28\end{array}$ & $\begin{array}{l}11.30^{b} \\
\pm 0.33\end{array}$ \\
\hline
\end{tabular}

*Values are represented as mean \pm SD.

*The treatment means were separated using Tukey HSD at $\mathbf{0 . 0 5 \%}$ probability level.

*Level not connected by same letter are significantly different in vertical columns. 
Table 9: Minimum Inhibitory Concentrations (MIC), Minimum Fungicidal Concentrations (MFC) and Minimum Bactericidal Concentrations (MBC) of different extract of $R$. roseum against different pathogens $(\mu \mathrm{g} / \mathrm{ml})$.

\begin{tabular}{|c|c|c|c|c|c|c|c|c|c|c|c|}
\hline \multirow{2}{*}{$\begin{array}{c}\text { Pathogen } \\
\text { Fungi }\end{array}$} & \multicolumn{2}{|c|}{ Petroleum ether } & \multicolumn{2}{|c|}{ Methanol } & \multicolumn{2}{|c|}{ Chloroform } & \multicolumn{2}{|c|}{ Etahnol } & \multicolumn{2}{|c|}{ Acetone } & \multirow{2}{*}{$\frac{\text { STANDARDS }}{\text { MIC (MBC/MFC) }}$} \\
\hline & MIC & $\begin{array}{l}\text { MBC/ } \\
\text { MFC }\end{array}$ & MIC & $\begin{array}{l}\text { MBC/ } \\
\text { MFC }\end{array}$ & MIC & $\begin{array}{l}\text { MBC/ } \\
\text { MFC }\end{array}$ & MIC & $\begin{array}{l}\text { MBC/ } \\
\text { MFC }\end{array}$ & MIC & $\begin{array}{l}\mathrm{MBC} / \\
\mathrm{MFC}\end{array}$ & \\
\hline S. rolfsii & 2.50 & 5.00 & 2.50 & 4.50 & 2.50 & 5.00 & 5.00 & 6.00 & 5.00 & 6.25 & $0.50(0.80)$ \\
\hline R. solani & 2.50 & 4.50 & 0.65 & 0.65 & 2.50 & 5.00 & 0.65 & 0.65 & 0.65 & 1.25 & $1.00(1.25)$ \\
\hline T. indica & 0.65 & 0.85 & 0.65 & 0.75 & 0.65 & 1.25 & 0.65 & 0.75 & 0.65 & 0.65 & $0.50(0.60)$ \\
\hline F. oxysporium & 1.25 & 1.25 & 0.65 & 0.75 & 0.65 & 0.65 & 1.25 & 1.75 & 1.25 & 1.75 & $0.50(0.60)$ \\
\hline \multicolumn{12}{|l|}{ Bacteria } \\
\hline P. multocida & - & - & - & - & 1.25 & 1.25 & 0.65 & 0.80 & 1.25 & 1.65 & $0.50(0.70)$ \\
\hline S. enterica & - & - & - & - & - & - & 1.25 & 1.25 & 2.50 & 2.50 & $0.25(0.25)$ \\
\hline M. plutonius & - & - & 2.50 & 3.00 & - & - & 1.50 & 1.50 & 1.25 & 2.50 & $0.65(0.80)$ \\
\hline X. oryzae & - & - & 2.50 & 2.75 & - & - & 2.50 & 3.00 & - & - & $0.65(0.75)$ \\
\hline
\end{tabular}

Chloramines $\mathbf{T}$ and Ampicillin are used as standards for fungi and bacteria respectively 
hanging drop method and comprising species to species interaction, type and amount of extract was attained. The highest antifungal activity was traced in 100 percent methanolic extract (Deora and Suhalka , 2017; Latinović et al., 2018). Another research revealed by Latinović et al. (2019) showed that, the extracts of three bryophyte species are confirmed to have suppressive effects on grey mould disease $(B$. cinerea). Methanol extracts of one leafy liverwort $(P$. platyphylla) and two mosses, one aquatic ( $C$. fontinaloides) and one terrestrial (A. viticulosus), were applied in vitro to $B$. cinerea, after which experiment confirmed inhibition of fungal growth was observed. Antifungal activities of methanol extract of the moss species Fontinalis antipyretica Hedw. var. antipyretica, Hypnum cupressiforme Hedw., and Ctenidium molluscum (Hedw.) Mitt., were also analyzed by Veljić et al., in 2009.

Effect of extracts of $R$. roseum were examined against the pathogenic plant bacteria

On comparing all undertaken extracts with positive control (ampicillin and streptomycin) significantly lower values were observed for Xanthomonas oryzae. Out of the six extracts (aqueous and organic) of $R$. roseum, the activity was maximum 12.31 with ethanol extract and minimum 10.22 with methanol extract (Table 5). No extract shows better activity when compared to standard antibiotics.

However, in case of Melissococcus plutonius out of the six extracts (aqueous and organic) of $R$. roseum, the activity was maximum 15.26 with acetone extract and minimum 6.37 with methanol extract (Table 6). As compared to standard antibiotics acetone extract shows good activity. In ethanol and acetone extract highly significant values were observed at $40 \mu \mathrm{g} \mathrm{ml}^{-1}$ conc. over both the positive control.

In addition, Pasteurella multocida out of the six extracts (aqueous and organic) of $R$. roseum, the activity was maximum 20.24 with ethanol extract and minimum 12.20 in acetone extract (Table 7). On comparing all undertaken extracts with positive control (ampicillin and streptomycin) significantly higher values were obtained in ethanol and acetone at all conc. except, at $500 \mu \mathrm{g} \mathrm{ml}^{-1}$ conc.

Further, in case of Salmonella enterica out of the six extracts (aqueous and organic) of $R$. roseum, the activity was maximum 16.26 and minimum 11.19 was observed with acetone extract (Table 8). No extract shows better activity when compared to standard antibiotics. Comparison of acetone and ethanol extract with positive both the controls showed significantly lower, but at the same time non significant values were observed at 200 and $500 \mu \mathrm{g} \mathrm{ml}^{-1}$ conc.

Dewanjee et al., (2007) showed that methanol extract of $D$. peregrina fruits (MEDP) and $C$. grandis leaves (MECG) had highest sensitivity against $E$ coli strains. MECG showed major activity against Staphylococcus aureus, E. coli, S. dysenteriae, $S$. soneii and $P$. aeruginosa; while resistant to $S$. flexneri and $S$. boydii. In the present study the strain of $E$. coli showed least sensitivity for the ethanol extract. Other results revealed by Abdel-Shafi et al., (2017), the pathogenic bacteria L. monocytogenes LMG 10470, E. coli LMG 8223, $B$. cereus ATCC 14579 and P. aeruginosa LMG 8029 were suppressed by the aqueous methanolic extracts of Imbibryum spp., B. convoluta and Trichostomum spp. The fusion of Imbibryum spp. extract and tetracycline has synergistic effects against $P$. aeruginosa while the mixing of Trichostomum spp. extract with tetracycline has antagonistic effect against $P$. aeruginosa. Off Petroleum ether, chloroform, benzene, methanolic and aqueous extracts of five ferns $A$. caudatum, $A$. evecta, $P$. confusa, $P$. argyraea and $L$. microphyllum against Xanthomonas campestris $p v$. centellae. The methanolic extracts of all the ferns showed successful antibacterial activity of against the performed bacteria. Phytochemical study of all the extracts showed that antibacterial activity and the MIC and RPI values, $A$. evecta could be used as potential plant for the supervision of pathogenic bacteria, Xanthomonas campestris $p v$. centellae which is known to cause diseases on various crops especially Centella asiatica (Gracelin et al., 2012). All of the tested outcomes confirmed that the aqueous methanolic extracts of selected bryophyte species are proven promising antibacterial agents however, Sabovljevic et al., in 2010 also showed that DMSO extracts of three selected bryophyte species, two mosses and a liverwort (A. undulatum (Hedw.) P. Beauv., M. polymorpha L. sp. ruderalis Bischl. and Boisselier, P. patens (Hedw.) Bruch and Schimp.) estimated the results by microdilution method against eight bacterial species. All examined extracts of bryophytes are confirmed to be active against all bacteria tested. 
Out of the six (different organic and aqueous) extracts of $R$. roseum ethanol and acetone extracts showed maximum inhibitory activity in $S$. rolfsii. In addition, maximum MIC value was observed 5.00 (acetone), whereas, MFC value as 6.25 (acetone) and $\mathrm{MBC}$ value as 3.00 (ethanol) in $X$. oryzae. Study also shows the greater MIC values with their respective values of $\mathrm{MFC} / \mathrm{MBC}$, this may be due to contaminated form of the bio-active compounds. However, in few results these values were found equal to their respective $\mathrm{MFC} / \mathrm{MBC}$ values, which may be due to presence of a specific group of compounds. Study carried out by Mewari and his coworkers (2008) recorded the higher values of $\mathrm{MBC} / \mathrm{MFC}$ in methanol extract over their MIC values, while, flavonoid showed the similar results of MIC and MBC/MFC.

The bioactivity data obtained from the present work points toward maximum antifungal activity in methanol, acetone and chloroform extracts of undertaken moss i.e. Rhodobryum roseum over other extracts. Furthermore, different extracts of aqueous and organic at different concentrations $\left(1000,500,200\right.$ and $\left.40 \mu \mathrm{g} \mathrm{ml}^{-1}\right)$ and at different time intervals $(24,48$ and $72 \mathrm{hrs})$ indicated maximum inhibition on the growth of $F$. oxysporum in acetone extract with $1000 \mu \mathrm{g} \mathrm{ml}^{-1}$ conc. at $24 \mathrm{hrs}$ and $T$. indica in chloroform extract with $1000 \mu \mathrm{g}$ $\mathrm{ml}^{-1}$ conc. at $24 \mathrm{hrs}$. However, in case of bacteria maximum inhibition was observed on the growth of P. multocida in ethanol extract with $1000 \mu \mathrm{g} \mathrm{ml}^{-1}$ conc., which may be due to contaminated form of bioactive compounds (Table 9). Results of few experiments show similar values of MIC and MBC/MFC which may be because of specific group of compound.

\section{Conclusion}

Above study concluded that, selected species of bryophyte i.e. $R$. roseum had bioactivity against selected test of bacteria and fungi. Bioactivity varied according to the solvent used, concentrations applied and time intervals. Hence, $R$. roseum products may be considered as bio-control representatives and play noteworthy roles for practical perspectives in a communally, economically and ecologically vigorous crop management structure.

\section{Acknowledgements}

The authors pay their gratitude to Prof A. K. Sharma and Dr. Rashmi Shrivastava, GBPUA\&T, Pantnagar for their support during different stages of work.

\section{References}

Abdel-Shafi, S., Hussein, Y., Lashin, G. and Abdel-Monaem, Al-S. 2017. An Evaluation of the Antibacterial and Antiviral Activities of Some Bryophytes. Egypt. $J$. Microbiol, 52(1):63 - 86 .

Basri, D. F. \& Fan, S. H. 2005. The potential of aqueous and acetone extracts of galls of Quercus fectoria as antibacterial agents. Indian Journal Pharmacology. 37: 26-29.

Chopra RS. 1975. Taxonomy of Indian mosses. Publication and information directorate (C S I R), New Delhi.

Deora, G. S. and Suhalka, D. 2017. Evaluation of Bryophyte for Green Fungicides as Alternative Treatment to Control Plant Pathogen. International Journal of Pharmacognosy and Phytochemical Research, 9(10): 1373-1379.

Dewanjee S, Kundu M, Maiti A, Majumdar R, Majumdar A and Mandal SC. 2007. In vitro evaluation of antimicrobial activity of crude extract from plants Diospyros peregrina, Coccinia grandis and Swietenia macrophylla. Trop. J. Pharma. Res. 6(3): 773.

Gangulee HC. 1969. Mosses of eastern India and adjacent regions. III Bull. Bot. Soc. Beng. 23: 131-134.

Gracelin, D.H.S., Britto, A.J.D. and Kumar, P.B.J.R.. 2012. Antibacterial screening of a few medicinal ferns against antibiotic resistant phyto pathogen. International journal of pharmaceutical sciences and research, 3(3): 868-873.

Janovska D, Kubikova K. \& Kokoška L. 2003. Screening for antimicrobial activity of some medicinal plants species of traditional Chinese medicine. Czech Journal of Food Sciences. 21(3): 107-10.

Kumar K, Singh KK, Nath V and Asthana AK. 2002. Concept of bryophyte in classical text of three species of bacteria. Appl. Boil. 18: 77.

Latinović, N., Sabovljević, M. S., Vujičić, M., Latinović, J. and Sabovljević, A. 2018. Searching on novel biofungicides from bryophytes. XI International Scientific Agriculture Symposium “Agrosym”, Jahorina October 04-07, 2018.

Latinović, N., Sabovljević, M. S., Vujičić, M., Latinović, J. and Sabovljević, A. 2019. Bryophyte extracts suppress growth of the plant pathogenic fungus Botrytis cinerea. Botanica Serbica, 43 (1): 9-12. 
Mekuria T, Blaeser P, Steiner U, Dehne HW, Lyr H, Ressell PE and Sisler HD. 1999. Bryophytes as a new source of antifungal substance in crop protection. Modern fungicides and anti fungal compounds II. 12 ${ }^{\text {th }}$ Int. Reinhardsbrunn Symposium, Fridrichorda, Thuringia, Germany: pp 483.

Mekuria T, Steiner U, Hindorf H, Frahm JP and Dehne HW. 2005. Bioactivity of bryophyte extracts against Botrytis cinerea, Alternaria solani and Phytophthora infestans. $\boldsymbol{J}$. Appl. Bot. Food Qual. 79: 89.

Mewari N, Chaturvedi P, Kumar P and Rao PB. 2007. Antimicrobial activity of moss extract against plant pathogens. J. myco. pl patho. 37(2): 359.

Sabovljevic A, Sokovic M, Glamoclija J, Ciric A, Vujicic M, Pejin B and Sabovljevic M. 2010. Comparison of extract bio-activities of in situ and in vitro grown selected bryophyte species. Afr. J. Microbio. Res. 4: 808.

Saxena DK, Singh S and Srivastava K. 2008. Taxonomy of Rhodobryum from Kumaon and Garhwal region of Uttarakhand, India. Ind. J. For. 31(3): 437.

Sengupta S, Ghosh SN and Das AK. 2008. Antimycotic potentiality of the plant extract Bacopa monnieri (L.) Penn. Res. J. Bot. 3(2): 83.

Smith AJE. 1978. The moss flora of Britain and Ireland. Cambridge University Press, London.

Veljić M, Djurić A, Soković M, Cirić A, Glamoćlija J and Marin PD. 2009. Antimicrobial activity of methanol extracts of Fontinalis antipyretica, Hypnum cupressiforme and Ctenidium molluscum. Arch. Biol. Sci.61: 225. 